\title{
Multi-scale MSDT inversion based on LAI spatial knowledge
}

\author{
ZHU XiaoHua $^{1,2}$, FENG XiaoMing $^{3 *} \&$ ZHAO YingShi $^{2}$ \\ ${ }^{1}$ Institute of Remote Sensing Applications, Chinese Academy of Sciences, Beijing 100101, China; \\ ${ }^{2}$ Graduate School of the Chinese Academy of Sciences, Beijing 100049, China; \\ ${ }^{3}$ Research Center for Eco-Environmental Sciences, Chinese Academy of Sciences, Beijing 100085, China
}

Received January 17, 2011; accepted May 13, 2011; published online December 15, 2011

\begin{abstract}
Quantitative remote sensing inversion is ill-posed. The Moderate Resolution Imaging Spectroradiometer at $250 \mathrm{~m}$ resolution (MODIS_250m) contains two bands. To deal with this ill-posed inversion of MODIS_250m data, we propose a framework, the Multi-scale, Multi-stage, Sample-direction Dependent, Target-decisions (Multi-scale MSDT) inversion method, based on spatial knowledge. First, MODIS images $(1 \mathrm{~km}, 500 \mathrm{~m}, 250 \mathrm{~m})$ are used to extract multi-scale spatial knowledge. The inversion accuracy of MODIS_1 $\mathrm{km}$ data is improved by reducing the impact of spatial heterogeneity. Then, coarse-scale inversion is taken as prior knowledge for the fine scale, again by inversion. The prior knowledge is updated after each inversion step. At each scale, MODIS_1 km to MODIS_250m, the inversion is directed by the Uncertainty and Sensitivity Matrix (USM), and the most uncertain parameters are inversed by the most sensitive data. All remote sensing data are involved in the inversion, during which multi-scale spatial knowledge is introduced, to reduce the impact of spatial heterogeneity. The USM analysis is used to implement a reasonable allocation of limited remote sensing data in the model space. In the entire multi-scale inversion process, field data, spatial knowledge and multi-scale remote sensing data are all involved. As the multi-scale, multi-stage inversion is gradually refined, initial expectations of parameters become more reasonable and their uncertainty range is effectively reduced, so that the inversion becomes increasingly targeted. Finally, the method is tested by retrieving the Leaf Area Index (LAI) of the crop canopy in the Heihe River Basin. The results show that the proposed method is reliable.
\end{abstract}

ill-posed inversion, prior knowledge, MSDT, multi-scale

Citation: Zhu X H, Feng X M, Zhao Y S. Multi-scale MSDT inversion based on LAI spatial knowledge. Sci China Earth Sci, 2012, 55: 1297-1305, doi: 10.1007/s11430-011-4312-0

The Leaf Area Index (LAI) is a key structural characteristic of vegetation and plays a significant role in global change research. Inversion based on a physical model is an important way to calculate the regional LAI. However, there are numerous unknown parameters in the model and remote sensing information is limited. Physical model inversion is an "underdetermined" process of deriving previously unknown information from limited prior information, representing an "ill-posed" problem [1]. This problem has aroused wide attention and has led to the recognition that a

*Corresponding author (email: fengxm@ rcees.ac.cn) priori knowledge is indispensable for its solution. In regional remote sensing inversion, it is difficult to obtain a wide range of observations, and prior information about complex surface processes is limited. Therefore, remote sensing inversion research has focused on how to make full use of prior knowledge and remote sensing observations. Qu et al. [2] proposed a hybrid inversion technique based on a Bayesian network for estimating land surface vegetation parameters from remotely sensed data. This method obtained dynamic a posteriori information on model parameters and achieved a more accurate retrieval of the LAI. Wang et al. [3] coupled a crop growth model to a radiative transfer model, using the Lagrangian to construct a cost 
function for solution optimization [4]. Through interaction among prior information with parameter adjustment, prior information on the surface LAI, and multi-temporal remotely sensed observations, the method produced optimum LAI profiles. Besides adding more information, the full use of various nonlinear inversion optimizations can also effectively avoid the problem caused by linearization of nonlinear problems [5-8]. At present, most inversion uses algorithms directly, which we call simplex inversion [9]. In this approach, there is less focus on rational use of prior knowledge, efficient allocation of observations, and state analysis of model parameters. Therefore, research on inversion methods is lacking. Because of the lack of prior knowledge and remote sensing data in regional large-scale remote sensing inversion, $\mathrm{Li}$ et al. $[10,11]$ stated that all parameters should not be retrieved from observations simultaneously and, taking the relationship between parameter sensitivity and model inversion into account, they proposed a Multistage, Sample-direction Dependent, Target-decisions (MSDT)-based, Uncertainty and Sensitivity Matrix (USM). This method is a knowledge- based inversion that deals with allocation of limited remote sensing information in model parameter space and its application to remote sensing inversion, allowing the information to be effectively allocated in a complex system. Many studies [9, 12, 13] have shown this inversion method to be more reliable than previous ones.

The ultimate goal of remote sensing inversion is its application to large-scale regions but, in regional applications, scale is another factor affecting the inversion. For example, because of the spatial heterogeneity of surface landscape structure, field data participates in the pixel-scale inversion, so the scale effect and scale change become important constraints [14-17]. Feng et al. [9] considered the spatial correlation of geographic data and proposed Multi-scale Analysis MSDT inversion based on Multi-resolution Analysis (MRA) of a wavelet transform, which extended the content of prior knowledge and took multi-scale spatial knowledge into account in a preliminary way. Although the scale effect restricts quantitative remote sensing inversion, if we can quantify the effect and put it to use effectively, it can provide useful prior knowledge for multi-scale retrieval of surface parameters.

This paper takes a MODIS_250m LAI inversion as a typical case to study the "ill-posed" problem of regional scale LAI inversion, in an experimental area within the agricultural region of the Heihe River. To reduce the uncertainty of model inversion, prior knowledge is introduced in two ways: (1) Spatial information is extracted from the spatial relationship among multi-scale data, and is used as prior knowledge that participates in the inversion; and (2) based on inversion strategy, field data is also used as prior knowledge in each scale inversion. Through USM analysis, the most uncertain parameters are inversed by the most sensitive data, which makes all the remote sensing observations participate in the inversion in stages, providing information across the entire inversion process. In the whole process of multi-scale inversion, field data, spatial knowledge and multi-scale remote sensing observations are involved. The final estimated LAI is a best result that contains all this information. This study attempts to find a solution for regional remote sensing inversion with an "ill-posed" problem.

\section{Methods}

\subsection{Canopy reflectance model}

The turbid medium, Markov chain canopy reflectance model (ACRM) incorporates Markov properties of stand geometry, making it applicable to plant canopies largely composed of vertical elements such as corn [18].

The ACRM model contains the PROSPECT leaf optical model [19], simulating the reflectance and transmittance from 400 to $2500 \mathrm{~nm}$ of a leaf, through the relationship function of leaf structure parameters and biochemical parameters. The model has a high computational efficiency and can explain the specular reflection of a leaf surface. The calculation of multiple scattering in the model is the same as in the SAIL model [20]. The ACRM model is a onedimensional, bidirectional turbid medium radiative transfer model that has been modified to take into account the "hot spot" and "leaf mirror reflection" effects of plant canopy reflectance [21]. Use of a turbid medium defines the canopy as a horizontally homogenous and semi-infinite layer, consisting of small vegetation elements that act as absorbing and scattering particles of given geometry and density. Given canopy structure and environmental parameters, the model can calculate canopy reflectance for any sun altitude and observation direction. The Price model [22] is incorporated in the ACRM to calculate soil reflectance. The soil reflectance spectra in the model are approximated as a function of four basis vectors, where the first two vectors explain $94.2 \%$ of spectral variability in soil reflectance. The simulation of canopy reflectance by the ACRM model depends neither on specific vegetation type nor environmental background. Consequently, the model is universally adopted for calculating vegetation canopy reflectance [20, 23, 24]. However, the model has many parameters, including external parameters $\left(\theta_{\mathrm{Z}}, \theta_{\mathrm{V}}, \beta, \varepsilon\right)$, canopy structural parameters (LAI, $s z, t h m)$, soil parameters $\left(s_{1}, s_{2}, s_{3}, s_{4}\right)$, leaf biochemistry parameters $\left(C_{\mathrm{ab}}, C_{\mathrm{m}}, C_{\mathrm{w}}, C_{\mathrm{bp}}, N\right)$ and so on.

\subsection{Spatial knowledge}

In this paper, spatial knowledge is defined as surface spatial heterogeneity information, describing the scale bias of different-resolution remote sensing data, expressed by the parameter "err".

Based on higher-resolution imagery, err $=\mathrm{LAI}_{\mathrm{p}}-\mathrm{LAI}_{\text {mean }}$ is defined as the bias for the LAI retrieved from up-scaling images, where $\mathrm{LAI}_{p}$ is the LAI retrieved from lower-resol- 
ution images, $\mathrm{LAI}_{\text {mean }}$ is the average of LAI retrieved from higher-resolution images (which is taken as relative truth), and err is the difference of $\mathrm{LAI}_{\mathrm{p}}$ and $\mathrm{LAI}_{\text {mean }}$. Inversion results from lower resolution images in the multi-scale MSDT inversion are corrected by err, via its use as spatial information. If LAI is directly calculated by the ACRM model $\rho=F\left(\phi\right.$, LAI, ALA, $\left.\cdots, N, C_{\mathrm{ab}}, C_{w}, \cdots, \rho_{s}\right)$, it would be an underdetermined result. The purpose here is to obtain scale information of different-resolution remote sensing data, so the semi-empirical conversion formula between LAI and the Normalized Difference Vegetation Index (NDVI) is established as follows $[25,26]$ :

$$
\begin{aligned}
\mathrm{LAI} & =f(\mathrm{NDVI}) \\
& =\frac{-1}{K_{\mathrm{NDVI}}} \log \left(\frac{\mathrm{NDVI}^{-} \mathrm{NDVI}_{\infty}}{\mathrm{NDVI}_{\mathrm{s}}-\mathrm{NDVI}_{\infty}}\right) \\
& =a \log (b \mathrm{NDVI}+c),
\end{aligned}
$$

where the extinction coefficient $K_{\mathrm{NDVI}}$ and $\mathrm{NDVI}_{\infty}$ are calculated by the SAIL model [20], $\mathrm{NDVI}_{\infty}$ is often close to the pure vegetation NDVI when the leaf angle distribution is spherical, $K_{\mathrm{NDVI}}$ is close to 0.5 [27], $\mathrm{NDVI}_{\mathrm{s}}$ is the NDVI of bare soil, and $a, b$, and $c$ are constants. The formula will be applied to higher-resolution remote sensing images.

Ignoring the nonlinear formula of NDVI, i.e. $\mathrm{LAI}_{p}$ $\approx f\left(\frac{1}{n} \sum_{i=1}^{n} \mathrm{NDVI}_{i}\right)=f(m \mathrm{NDVI}) \quad[28], m \mathrm{NDVI}$ is the mean value of the higher-resolution $n$-pixel NDVI. Then $\mathrm{LAI}_{\text {mean }}=\frac{1}{n} \sum_{i=1}^{n} f(\mathrm{NDVI})$ is approximated by a secondorder Taylor development of $f$ around $\left(\mathrm{NDVI}_{i}=m \mathrm{NDVI}\right)$. Finally, multi-scale deviation formulas are shown below, with more details available in document [29]:

$$
\begin{aligned}
e r r & =-\frac{1}{2} \sigma_{\mathrm{NDVI}} f^{\prime \prime}(m \mathrm{NDVI}) \\
& =-\left.\frac{1}{2} \sigma_{\mathrm{NDVI}}(a \log (b \mathrm{NDVI}+c))^{\prime \prime}\right|_{\mathrm{NDVI}=m \mathrm{NDVI}} \\
& =\frac{a b^{2} \sigma_{\mathrm{NDVI}}}{2(b m \mathrm{NDVI}+c)^{2}},
\end{aligned}
$$

where $m$ NDVI, $\sigma_{\mathrm{NAVI}}$ are mean and variance of higherresolution $n$-pixel NDVI; $a, b, c$ are the constants.

The spatial information parameter err, established by semi-empirical formulae, is affected by the accuracy of the statistical model and remainder term of Taylor expansion. Since our intent is to introduce spatial knowledge into the multi-scale inversion strategy, the experience factors of eq. (1) are obtained by physical model simulation and eq. (1) is a formula for extracting spatial knowledge and not for calculating the LAI. Therefore, the final result err is only relevant to the mean and variance of the NDVI.

\subsection{Uncertainty and sensitivity matrix}

The Uncertainty and Sensitivity Matrix (USM) describes parameter sensitivity to each given direction/band of observations. During the inversion, the data to be used and the parameters to be inversed are decided by the USM. The most sensitive observation data would be used to calculate the most uncertain parameters step by step, finally realizing Multi-stage, Sample-direction Dependent, Target-decisions (MSDT) inversion. The USM is defined as [30]:

$$
\operatorname{USM}(i, j)=\frac{\Delta \rho(i, j)}{\rho_{\text {exp }}(i)},
$$

where $\Delta \rho(i, j)$ is the difference between the maximum and minimum $\rho$ on the $i$ th direction/band while the $j$ th parameter varies in its uncertainty range, and other parameters are fixed as best guesses; $\rho_{\exp }(i)$ is the $\rho$ on the $i$ th direction/band when all the parameters are fixed as best guesses. MSDT, based on the USM, provide a more objective model inversion process [30].

\subsection{Multi-scale MSDT based on LAI spatial knowledge}

There are only a small number of MODIS_250m bands, but many model parameters. To accurately estimate LAI, we should make full use of MODIS data. MODIS_250m provides the main image and MODIS_1 km and MODIS-500m are used as auxiliary data. These are combined with spatial knowledge and measured surface data to carry out the multi-stage, multi-scale LAI inversion.

As shown in Figure 1, field data is introduced into the inversion process as prior knowledge and the scale information "err" derived by eq. (2); that is, the difference between the inversion results of MODIS_1 $1 \mathrm{~km}$ and MODIS_500m is used as spatial knowledge. Then, according to the USM matrix, the most sensitive observations are gradually introduced into the MODIS_1 km inversion process, adjusting and updating the most uncertain parameters in stages. Model parameter information (mean and variance) is updated once, after the first scale MSDT inversion. Information is updated from interaction among field data, scale information (spatial knowledge) and MODIS remote sensing observations. This updated information continues to participate in the MSDT inversion, being used as new prior knowledge for the next scale. Then the remaining operations were treated analogously until the last scale. Finally, all remote sensing observations are introduced stepwise to multistage target-decisions by USM analysis, providing information for parameter updates. Simultaneously, spatial knowledge is gradually introduced to reduce the effect of spatial heterogeneity during the LAI inversion.

From MODIS_1 km to MODIS_250m, all remote sensing observations and scale information have been fully used, and multi-scale inversion is refined progressively by pa- 


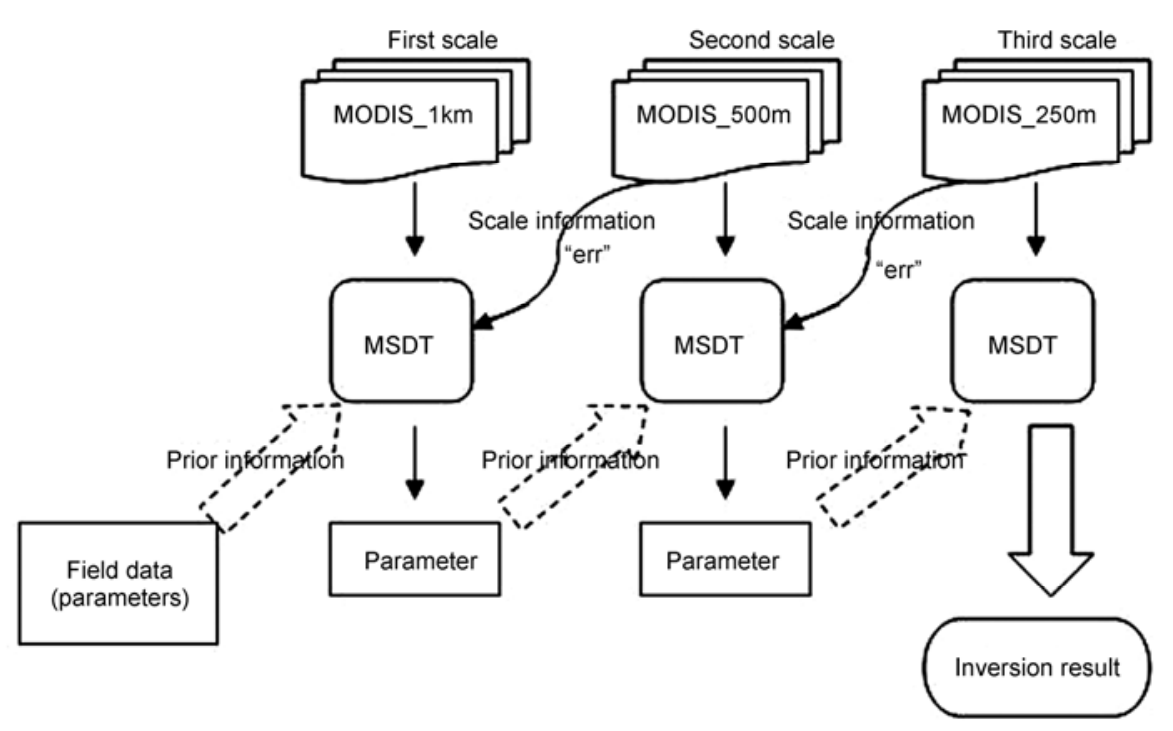

Figure 1 Flows of multi-stage, multi-scale LAI inversion.

rameter updates. In each scale multi-stage inversion, the data to be used and the parameters to be inversed are decided by the USM. The USM analysis prescribes that fewer sensitive and uncertain parameters are inversed with each stage, so the inversion process of each scale is relatively stable. According to Tarantola "inverse problem theory" [31] and an assumption that observations and parameters obey a Gaussian distribution, the multi-scale cost function is [9, 12]:

$$
\begin{aligned}
E(X)= & \left(X-X^{\text {prior }}\right)^{T} C_{X}^{-1}\left(X-X^{\text {prior }}\right) \\
& +\int_{c}\left(\operatorname{LAIr}_{c}-\mathrm{LAI}_{c}^{\text {prior }}\right)^{T} C_{\mathrm{Lc}^{-1}}\left(\operatorname{LAIp}_{c}-\mathrm{err}-\mathrm{LAI}_{c}^{\text {prior }}\right) \mathrm{d} c \\
& +\int_{c}\left(d_{c}^{\mathrm{cal}}-d_{c}^{\mathrm{obs}}\right)^{T} C_{D}^{-1}\left(d_{c}^{\mathrm{cal}}-d_{c}^{\mathrm{obs}}\right) \mathrm{d} c
\end{aligned}
$$

where $X$ is the model parameters, $X^{\text {prior }}$ is the prior estimate of parameters, and $C_{X}$ is the corresponding prior covariance matrix. $\mathrm{LAIr}_{c}$ is the $c$-scale LAI value corrected by "err", $\mathrm{LAIr}_{c}=\mathrm{LAI}_{\mathrm{P} c}-\mathrm{err}_{c}, \mathrm{LAI}_{\mathrm{c}}$ prior is the prior value of $\mathrm{LAI}, C_{\mathrm{Lc}}$ is prior variance of the $c$-scale LAI. $d_{c}^{\text {cal }}$ is a modelcalculated result that contains $c$-scale spatial knowledge: $\rho=F\left(\phi, \operatorname{LAIr}_{c}, \operatorname{ALA}, \cdots, N, C_{\mathrm{ab}}, C_{w}, \cdots, \rho_{s}\right), \quad d_{c}^{\text {cal }}$ are $c$-scale observations, $C_{D}$ is the covariance matrix of observations. The covariance matrix of the first scale is calculated based on field data and is adjusted in each stage. Take $C_{X}$ for example,

$$
C_{X}=\left[\begin{array}{cccc}
\sigma_{1} & \sigma_{12} & \ldots & \sigma_{1 n} \\
\sigma_{21} & \sigma_{2} & \ldots & \sigma_{2 n} \\
\ldots & \ldots & & \ldots \\
\sigma_{n 1} & \sigma_{n 2} & \ldots & \sigma_{n}
\end{array}\right]=\left[\sigma_{i j}\right]_{i, j=1,2, \ldots n},
$$

$\sigma_{i j}$ is the covariance of parameter $i$ and $j$ when $(i \neq j)$, or is the parameter variance when $(i=j)$, and $n$ is the number of parameters that participate in the inversion. The covariance matrix of a later scale is calculated from the previous scale inversion results (mean and variance). Covariance matrix $C_{X}$ describes the uncertainty of parameters $X$.

To improve computational efficiency during each scale inversion, the maximum and minimum of the $80 \%$ confidence interval of parameters calculated at the end of each stage inversion are used as the parameter uncertainties for the next stage. The uncertainties of parameters not inversed are not changed. Then the USM is recalculated to choose the set of parameters for the next stage inversion, until completion of the multi-stage inversion. During the entire inversion process, parameter values from the previous scale serve as prior knowledge for the later scale and, with the introduction of observations and spatial information, the inversion is gradually refined to get a $250 \mathrm{~m}$ resolution LAI image.

\section{Experiment analysis and results}

MODIS data from July 1, 2008 in the Heihe River Basin was selected, all of which were subjected to radiation, geometric and atmospheric corrections. Corresponding field data was provided by the "Heihe experiment". During June and July, 2008, the experiment collected some information of vegetation canopy and leaf and measured soil reflectance at Yingke Oasis. Sample points are shown in Figure 2(a). The LAI collection method is as follows. Lengths and widths of sample leaves were measured manually and by LAI-2000 and LAI-3000 instruments. Correction factors for each crop were calculated by comparing the manual measured value and the instrument one (after conversion). Crop 
LAIs were obtained through multiplication of crop leaf length and width by the correction factors. The quadrat size in Yingke Oasis is either $180 \mathrm{~m} \times 180 \mathrm{~m}$ or $240 \mathrm{~m} \times 240 \mathrm{~m}$, but the resolution of MODIS is too low to match these sizes, so expectations during inversion are set by the average of field data within a MODIS pixel. To reduce bias in the inversion result caused by the narrow range of parameter uncertainties, these uncertainties are set by the union of boundary values within the study area and \pm 1 standard deviation of the average values within the sample area, that is, $\left(x_{i} \pm \sigma_{i}\right) \mathrm{U}\left(\min \_x_{i}, \max \_x_{i}\right)$.

Finally, the expected value and uncertainty of model parameters are shown in Tables 1 and 2. Some of these values are set by referring to previous studies [18, 22, 32, 33]. For example, the Angstrom turbidity factor $\beta$ is calculated by MODIS aerosol optical thickness based on an Angstrom turbidity formula [34], and "hot spots" are calculated by the function $\varepsilon=0.5 / \mathrm{LAI}[35]$.

Table 1 shows the expectations and uncertainties of vegetation canopy structure and leaf biochemical parameters; Table 2 shows the expectations and uncertainties of soil parameters. The weight coefficients of the four vector basic function $\varphi_{i}(\lambda)$ in Price's soil reflectance formula $G_{\text {soil }}(\lambda)=s_{1} \varphi_{1}(\lambda)+s_{2} \varphi_{2}(\lambda)+s_{3} \varphi_{3}(\lambda)+s_{4} \varphi_{4}(\lambda)$ are $s_{1}, s_{2}, s_{3}$, and $s_{4}$. Given the conditions of Tables 1 and 2, eq. (3) is used to obtain the USM, which is shown in Table 3.

Table 3 shows that Band 2 has the most sensitive observation data for LAI (the maximum value in the matrix). Therefore, we use this band (858 nm) (Band 2) to inverse the LAI and reduce its uncertainty. After this reduction, we use $412 \mathrm{~nm}, 1240 \mathrm{~nm}$, and $678 \mathrm{~nm}$ to inverse $C_{\mathrm{ab}}, s_{1}$, and $s z$, respectively, and reduce their corresponding uncertainties.

Table 1 Vegetation parameters

\begin{tabular}{|c|c|c|c|c|}
\hline Sign & Means & Expectations & Uncertainty & Data source \\
\hline LAI & Leaf area index & 2.5 & $0-5$ & Heihe Experiment \\
\hline$s z$ & Markov parameter & 0.7 & $0.4-1.0$ & {$[18,32,33]$} \\
\hline thm & Modal leaf angle $\left(^{\circ}\right)$ & 57 & $10-90$ & Heihe Experiment \\
\hline$C_{\mathrm{ab}}$ & Chlorophyll $\mathrm{a}+\mathrm{b}$ content $\left(\mu \mathrm{g} / \mathrm{cm}^{2}\right)$ & 50 & $25-80$ & Heihe Experiment \\
\hline$C_{\mathrm{m}}$ & Dry matter content $\left(\mathrm{g} / \mathrm{m}^{2}\right)$ & 50 & $20-100$ & Heihe Experiment \\
\hline$C_{\mathrm{w}}$ & Leaf equivalent water thickness $(\mathrm{cm})$ & 0.02 & $0.01-0.04$ & Heihe Experiment \\
\hline$N$ & Leaf structure parameter & 1.6 & $1.4-2.3$ & {$[18,32,33]$} \\
\hline
\end{tabular}

Table 2 Soil parameters

\begin{tabular}{ccccc}
\hline Sign & $s_{1}$ & $s_{2}$ & $s_{3}$ & $s_{4}$ \\
\hline Expectations & 0.2118 & 0.0616 & -0.0061 & -0.0293 \\
Uncertainty & $0.05-0.4$ & $-0.1-0.1$ & $-0.05-0.05$ & $-0.04-0.04$ \\
\hline
\end{tabular}

Table 3 Uncertainty and Sensitivity Matrix (USM), based on Tables 1 and 2

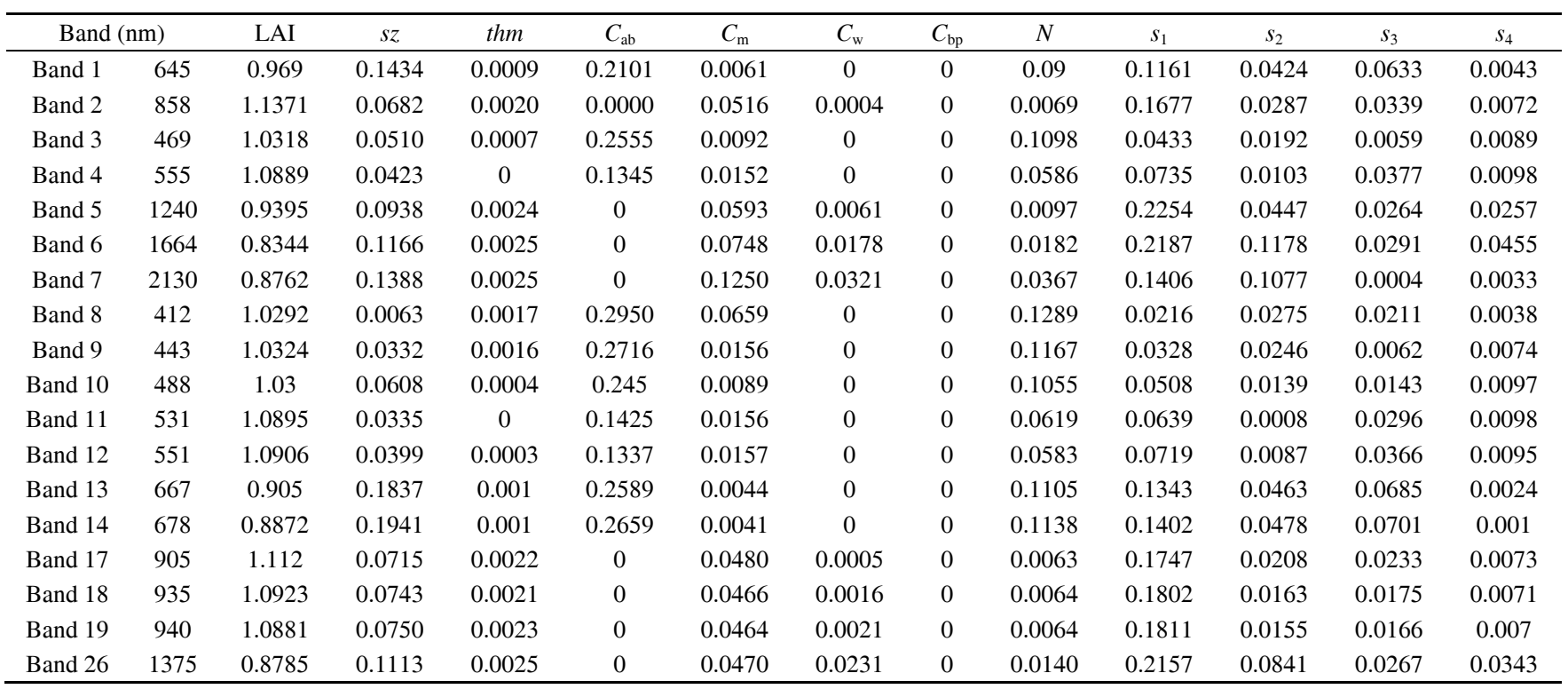


According to the stage inversion results, the USM is then recalculated to select the most uncertain parameters and observation data for the inversion. The USM matrix, parameters and observations are constantly adjusted during the stage inversion [30].

MSDT inversion is carried out on pixels. We use the results of the first scale as the expectations and parameter uncertainties for the second scale. As mentioned above, during second scale inversion, we also use the USM to decide the parameters to be inversed and the observations to involve. For the second scale, the data comes from MODIS_500m. Now, $s_{3}$ and $s_{4}$ in the Price soil reflectance formula are set to zero and $s_{2}$ is tied to $s_{1}$, which explains most of the spectral variability in the soil reflectance [22]. Thus, parameters $s_{2}, s_{3}, s_{4}$ are fixed during the second scale inversion and the remaining number of parameters is only nine. At this point, total number of observations is still less than that of the parameters, and the model cannot be divided into several sub-models that do not intersect in parameter sets or data sets. However, as long as the number of observations can be greater than or equal to the number of parameters ( $k \geqslant n, k \in K, n \in N)$ in each sub-model inversion, we can do an inversion of subset $X_{1}$. Then, selecting the new subset $X_{2}$ by recalculating the USM, we can repeat the above step until all the parameters are inversed [13]. During the third scale inversion, we need to fix some parameters. The setting of $C_{\mathrm{w}}$ is not important, because leaf water has no effect on reflectance in the visible and near-infrared wave bands [36]. The effect of $C_{\mathrm{m}}$ is predominantly in wavelengths longer than $1200 \mathrm{~nm}$ [37]; $C_{\mathrm{bp}}$ appears when leaves senesce and it represents light absorption by

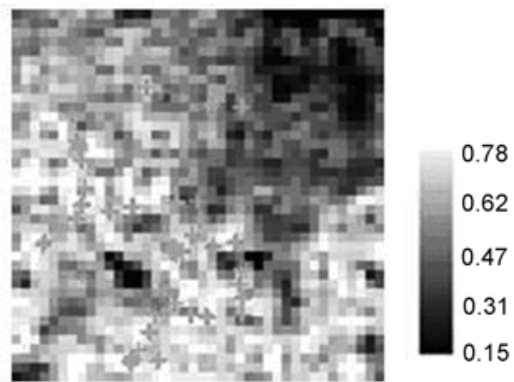

(a)

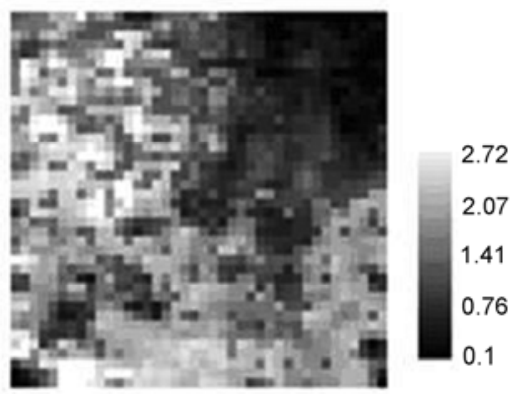

(c) non-chlorophyll pigments [36]. The vegetation in the study area consists of mature crops and the data comes from MODIS_250m, so we fix the three previously mentioned parameters. In each stage, the number of observations is greater than or equal to the number of parameters $(k \geqslant n$, $k \in K, n \in N$ ), and we split the model into sub-models accordingly. Only one or two parameters are inversed in each stage, until the inversion process is finished.

\subsection{Multi-scale MSDT inversion analysis}

This section validates the result of the multi-scale MSDT inversion (Figure 2(d)) and compares it with direct inversion (Figure 2(b)) and MSDT inversion (Figure 2(c)).

Figure 2(a) shows the NDVI in the study area (with location of LAI sample points); (b) is the direct inversion result, with model parameters set by field data directly; (c) is the MSDT inversion result, based on the USM, with parameters updated in stages; (d) is the MSDT inversion result based on spatial knowledge, whereby the method is integrated with multi-resolution scale information and is refined scale by scale. We used ground truth data to verify the accuracy of the different inversion methods. Because of the low resolution of MODIS, we averaged the field data within a MODIS pixel as "ground truth data". Validation results are shown in Figure 3.

As shown in Figures 2 and 3, the value of LAI retrieved by direct inversion (Figure 2(b)) is less than the measured LAI and the validation accuracy is about 0.56 (Figure 3(a)). The reason for this result is as follows. Direct inversion does not consider the impact of parameters on the model

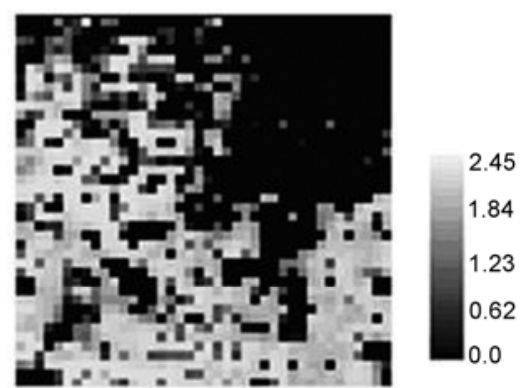

(b)



(d)

Figure 2 Results of variable inversion methods (resolution is $250 \mathrm{~m}$ ). (a) NDVI; (b) direct inversion; (c) MSDT inversion; (d) MSDT inversion based on spatial knowledge. 

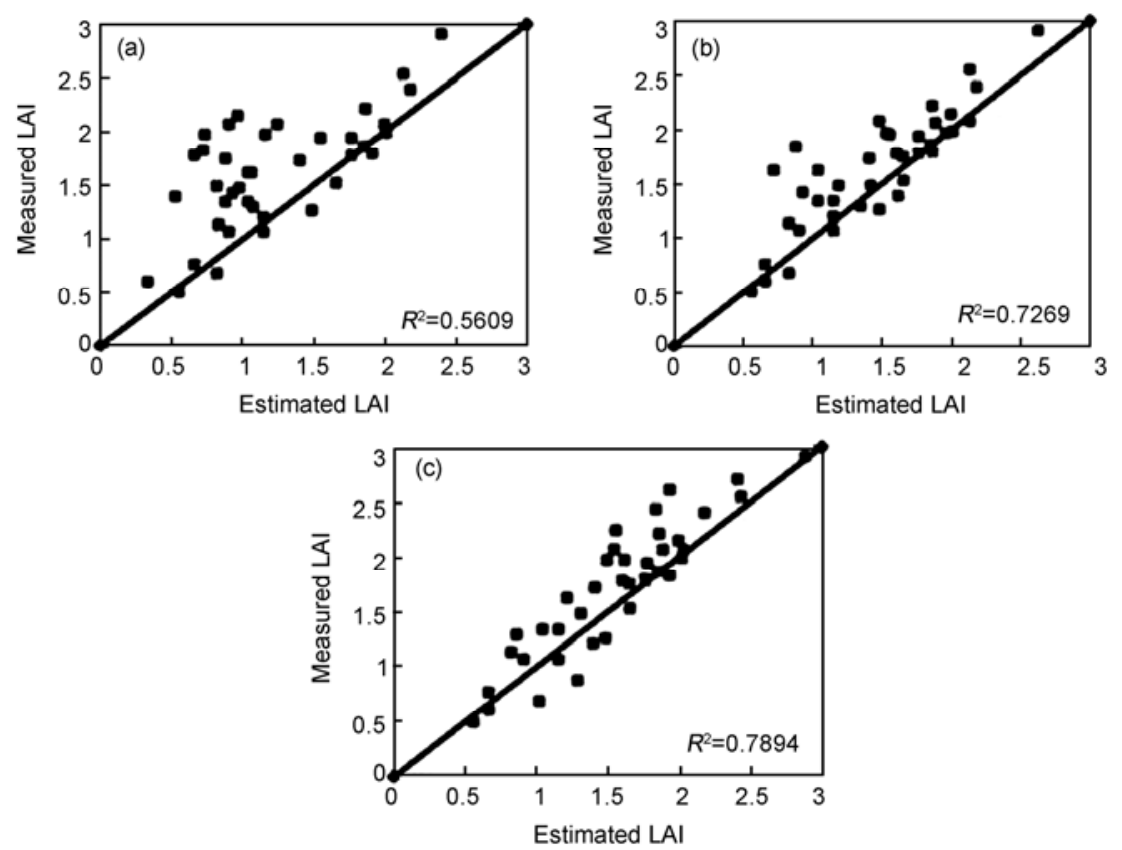

Figure 3 Ground validation of various inversion methods (resolution is $250 \mathrm{~m}$ ). (a) Direct inversion; (b) MSDT inversion; (c) MSDT inversion base on spatial knowledge.

and parameter uncertainties are set globally and not updated with each step. When there are fewer observations, it is likely to cause calculation failure of some insensitive parameters, indirectly leading to an inversion bias of sensitive parameters. Then, model parameters will converge to a local optimal value, causing a failure of the inversion process. In contrast, MSDT inversion (Figure 2(c)) is based on the USM, parameters converge in stages, and the result is more stable and reliable than direct inversion. There is also bias of the inversion result (Figure 3(b)), however, because the limited spectral information of MODIS_250m and fewer observations will affect the USM matrix pattern, reducing the probability of parameter convergence to the true value. This situation will cause error separation of parameters sets and data sets, leading to said estimation bias. Spatial knowledge and multi-scale remote sensing information are introduced in the method of Figure 2(d). The application of spatial information reduces the impact of surface spatial heterogeneity on coarse-scale inversion, making those inversion results more accurate as prior knowledge for finerscale inversion. Consequently, multi-scale, multi-level inversion produces a better result than the first two inverson types, with validation accuracy about 0.79 (Figure 3(c)).

LAI scale information extracted by multi-resolution MODIS data is introduced into MSDT inversion as prior knowledge, which improves the estimation accuracy of regional surface parameters. Meanwhile, the use of multiscale, multi-level calculation in the inversion process makes up for limited spectral information, accumulating prior information in several ways and focusing knowledge, thereby improving inversion accuracy. Based on the fusion of spa- tial knowledge and multi-scale spectral information, MODIS can be better applied to regional LAI estimation.

\section{Discussion}

Traditional LAI inversion methods are more concerned with algorithms rather than inversion strategy. There is also need for in-depth study of efficient allocation of observations and state analysis of model parameters. While there is an awareness of the advantages of multi-source data synergistic inversion, there are fewer studies on the application of spatial knowledge derived from multi-scale data.

In this study, we take full account of spatial information, remote sensing and field data. Region-wide field data is introduced to retrieve the LAI from MODIS in the form of mean and variance as described in section 2, which solves the mismatch problem of "point" data and "surface" pixel. Then, we use the multi-resolution data to synergistically inverse the LAI. Parameters retrieved from lower resolution data are corrected by spatial information calculated from higher-resolution data, and those parameters are used as prior knowledge in higher-resolution inversion, so that information is accumulated and more focused during the inversion process. During each scale inversion, we use the USM to analyze the status of model parameters and retrieve each uncertain parameter from sensitive data at each stage, making the allocation of remote sensing observations more rational and the inversion process more targeted. Experimental results show that the accuracy of multi-stage inversion is improved by $26 \%$, when compared to simple direct 
inversion. The possible reason for this improvement is that the physical model is usually nonlinear and high-dimensional, that direct inversion takes no account of the impact of sensitive parameters on the model, and that the result easily converges to a local optimum value [5]. MSDT based on the USM is an inversion method that is established on knowledge. Considering the distribution of limited information in model parameter space and application of such information during remote sensing inversion, and the stage wise calculation of parameters, MSDT is a more objective inversion method [9, 13].

When field data participates in the pixel-scale inversion, the inversion process will be constrained by the scale effect and scaling [14]. For example, with MODIS, there is a mismatch problem between field data and a MODIS pixel because field data is "point" in scale and a MODIS pixel is "surface" in scale. The scale effect of multi-source data is also a constraint for multi-scale data synergistic inversion, and there is a big deviation of LAI values retrieved by different methods [15]. Here we use the multi-scale inversion bias to extract spatial knowledge. As shown as eq. (3), we use NDVI variance of fine-scale and second order derivative of the correlation function to adjust the inversion result on a coarse scale, reducing the impact of surface spatial heterogeneity. Figure 3 indicates that accuracy of multistage inversion based on spatial knowledge is improved by about $7 \%$ compared to simple multi-stage inversion, and mean analysis shows that the inversion error is reduced by about $30 \%$. Because of homogeneous land cover in this case, the improvement of accuracy is not obvious. However, for a pixel of $50 \%$ vegetation coverage, its ground truth LAI value is 1.4 , its multi-stage inversion value is 0.89 , and the error is reduced by about $51 \%$, consistent with previous studies [28, 29].

The example MODIS_250m inversion illustrates that analysis of model parameter status, allocation of observations, and full use of prior knowledge, helps solve the "ill-posed" problem. That is, the strategy of remote sensing inversion and integration of ground information and LAI spatial knowledge is useful to improve overall inversion accuracy. There is still much need for future study, however.

\section{Conclusions}

To aid a solution to the "ill-posed" inversion problem, our study introduces the strategy of spatial knowledge and multi-scale inversion for LAI estimation, as follows. (1) Field data participate in the pixel inversion process in the form of expectation and variance, which solves the mismatch between field point data and MODIS pixels; (2) spatial knowledge extracted from multi-scale data is used to reduce the impact of surface spatial heterogeneity during inversion; and (3) the most sensitive data and the most uncertain parameters are decided by the USM; the most uncertain pa- rameters are inversed by the most sensitive data, which makes the inversion more targeted. The LAI retrieved for MODIS_250m is a combined result of remote sensing observations, field data and multi-scale spatial knowledge. Verification shows that the accuracy of estimated LAI can be improved over direct inversion from 0.56 to 0.79 , indicating that the inversion method is more accurate and reliable than traditional ones.

Our study has some limitations because when we analyze the scale effect, we only consider the influence of scale information on the LAI. We should also take into account the influence of scale information on the entire model parameter space. Because of the lack of remote sensing data, some parameters were fixed during the inversion; we therefore need more data for future research.

In short, this is a preliminary study of the quantitative remote sensing, "ill-posed" inversion problem, and there are still many scientific issues to be explored by future research.

This work was supported by Action Plan for West Development Program of the Chinese Academy of Sciences (Grant No. KZCX2-XB2-09), National Basic Research Program of China (Grant No. 2007CB714407) and National Natural Science Foundation of China (Grant No. 40801070).

1 Atzberger C. Object-based retrieval of biophysical canopy variables using artificial neural nets and radiative transfer models. Remote Sens Environ, 2004, 93: 53-67

2 Qu Y H, Wang J D, Wan H W, et al. A Bayesian network algorithm for retrieving the characterization of land surface vegetation. Remote Sens Environ, 2008, 112: 613-622

3 Wang D W, Wang J D, Liang S L. Retrieving crop leaf area index by assimilation of MODIS data into crop growth model. Sci China Ser D-Earth Sci, 2010, 53: 721-730

4 Kalnay E. Atmospheric Modeling, Data Assimilation and Predictability. London: Cambridge University Press, 2002

5 Wang L Y. Research on the treatment Methods of un-uniqueness in geodetic inversion. Master's Dissertation. Hunan: Central South University, 2008

6 Anderssen R S, Worthington M H, Cleary J R. Density modelling by Monte Carlo Inversion-I. Methodology. Geophy J Roy Astr Soc, 1972, 29: 433-444

7 Kirkpatrick S, Celatt C D, Vecchi M P. Optimization by simulated annealing. Science, 1983, 220: 671-680

8 Holland J H. Adaptation in Natural and Artificial Systems. Michigan: University of Michigan Press, 1975

9 Feng X M, Zhao Y S. On MSDT inversion with multi-angle remote sensing data. Sci China Ser D-Earth Sci, 2007, 50: 422-429

10 Li X W, Wang J D, Hu B X, et al. On utilization of a prior knowledge in inversion of remote sensing models. Sci China Ser D-Earth Sci, 1998, 28: 67-72

11 Goel N S, Thompson R L. Inversion of vegetation canopy reflectance model for estimating agronomic IV: Total Inversion of the SAIL Model. Remote Sens Environ, 1984, 15: 237-253

12 Gao F, Li X W, Xia G Z, et al. Multi-stage uncertainty inversion of multi-angle remote sensing based on prior information. Sci China Ser D-Earth Sci, 1998, 28: 346-350

13 Yan G J. Inversion studies on key parameters in remote sensing over land surface. Dissertation for the Doctoral Degree. Beijing: Institute of Remote Sensing Application, CAS, 1999

14 Su L H, Li X W, Huang Y X. An review on scale in remote sensing. Adv Earth Sci, 2001, 16: 544-548 
15 Tian Y H, Woodcock C E, Wang Y J. Multi-scale analysis and validation of the MODIS LAI product, I. Uncertainty assessment. Remote Sens Environ, 2002, 83: 414-430

16 Wang Y J, Woodcocka C E, Buemanna W. Evaluation of the MODIS LAI algorithm at a coniferous forest site in Finland. Remote Sens Environ, 2004, 91: 114-127

17 Yao Y J, Liu Q, Liu Q H, et al. LAI inversion uncertainties in heterogeneous surface. J Remote Sens, 2007, 11: 763-770

18 Kuusk A. A two-layer canopy reflectance model. J Quant Spectr Radiat Trans, 2001, 71: 1-9

19 Jacquemoud S, Baret F. PROSPECT: A model of leaf optical properties spectra. Remote Sens Environ, 1990, 34: 75-91

20 Verhoef W. Light scattering by leaf layers with application to canopy reflectance modeling: The SAIL model. Remote Sens Environ, 1984, 16: $125-141$

21 Kuusk A. The hot spot effect of a uniform vegetative cover. Soviet J Remote Sens, 1985, 3: 645-658

22 Price J C. On the information content of soil reflectance spectra. Remote Sens Environ, 1990, 33: 113-121

23 Baret F, Jacquemoud S, Guyot G, et al. Modeled analysis of the biophysical nature of spectral shifts and camparison with information content of broad bands. Remote Sens Environ, 1992, 41: 133-142

24 Feng X M, Zhao Y S. A spectral-directional reflectance remote sensing model of the semiarid landscape. J Remote Sens, 2005, 9: 337-342

25 Baret F, Guyot G. Potentials and limits of vegetation indices for LAI and FAPAR assessment. Remote Sens Environ, 1991, 35: 161-173

26 Garrigues S, Allard B D, Baret F. Influence of landscape spatial heterogeneity on the nonlinear estimation of leaf area index from moderate spatial resolution remote sensing date. Remote Sens Environ, 2006, 105: 286-298

27 Zhang R H, Sun X M, Su H B, et al. Remote sensing and scale transfering of levity parameters on earth surface. Remote Sens Land
Resour, 1999, 3: 51-59

28 Zhang W C, Zhong S, Hu S Y. Spatial scale transferring study on Leaf Area Index derived from remotely sensed data in the Heihe River Basin, China. Acta Eco Sin, 2008, 28: 2495-2503

29 Zhu X W, Feng X M, Zhao Y S, et al. Scale effect and error analysis of crop LAI inversion. J Remote Sens, 2010, 14: 586-599

30 Li X W, Gao F, Wang J D, et al. Uncertainty and sensitivity matrix of parameters in inversion of physical BRDF model. J Remote Sens, 1997, 1: 5-14

31 Tarantola A. Inversion Problem Theory: Methods for Data Fitting and Model Parameter Estimation. New York: Elsevier Science Publishing Company INC, 1987

32 Houborg R, Boegh E. Mapping leaf chlorophy mapping leaf chlorophyll and leaf area index using inverse and forward canopy reflectance modeling and SPOT reflectance data. Remote Sens Environ, 2008, 112: 186-202

33 Houborg R, Anderson M, Daughtry C. Utility of an image-based canopy reflectance modeling tool for remote estimation of LAI and leaf chlorophyll content at the field scale. Remote Sens Environ, 2009, 113: 259-274

34 Iqbal H. An Introduction to Solar Radiation. New York: Academic Press, 1983

35 Verhoef W, Bach H. Simulation of hyperspectral and directional radiance images using coupled biophysical and atmospheric radiative transfer models. Remote Sens Environ, 2003, 87: 23-42

36 Houborg R, Soegaard H, Boegh E. Combining vegetation index and model inversion methods for the extraction of key vegetation biophysical parameters using Terra and Aqua MODIS reflectance data. Remote Sens Environ, 2007, 106: 39-58

37 Botha, E J, Leblon, B, Zebarth B, et al. Non-destructive estimation of potato leaf chlorophyll from canopy hyperspectral reflectance using the inverted PROSAIL model. Int J Appl Earth Obs, 2007, 9: 360-374 